\title{
Optimal MRI Sequence for Identifying Occlusion Location in Acute Stroke: Which Value of Time-Resolved Contrast-Enhanced MRA?
}

\author{
A. Le Bras, H. Raoult, J.-C. Ferré, T. Ronzière, and J.-Y. Gauvrit
}

\begin{abstract}
BACKGROUND AND PURPOSE: Identifying occlusion location is crucial for determining the optimal therapeutic strategy during the acute phase of ischemic stroke. The purpose of this study was to assess the diagnostic efficacy of MR imaging, including conventional sequences plus time-resolved contrast-enhanced MRA in comparison with DSA for identifying arterial occlusion location.
\end{abstract}

MATERIALS AND METHODS: Thirty-two patients with 34 occlusion levels referred for thrombectomy during acute cerebral stroke events were consecutively included from August 2010 to December 2012. Before thrombectomy, we performed 3T MR imaging, including conventional 3D-TOF and gradient-echo T2 sequences, along with time-resolved contrast-enhanced MRA of the extra- and intracranial arteries. The 3D-TOF, gradient-echo T2, and time-resolved contrast-enhanced MRA results were consensually assessed by 2 neuroradiologists and compared with prethrombectomy DSA results in terms of occlusion location. The Wilcoxon test was used for statistical analysis to compare MR imaging sequences with DSA, and the $\kappa$ coefficient was used to determine intermodality agreement.

RESULTS: The occlusion level on the 3D-TOF and gradient-echo T2 images differed significantly from that of DSA $(P<.001$ and $P=.002$, respectively), while no significant difference was observed between DSA and time-resolved contrast-enhanced MRA $(P=.125) . \kappa$ coefficients for intermodality agreement with DSA ( $95 \% \mathrm{Cl}$, percentage agreement) were $0.43(0.3 \%-0.6 ; 62 \%), 0.32(0.2 \%-0.5 ; 56 \%)$, and 0.81 (0.6\%-1.0; 88\%) for 3D-TOF, gradient-echo T2, and time-resolved contrast-enhanced MRA, respectively.

CONCLUSIONS: The time-resolved contrast-enhanced MRA sequence proved reliable for identifying occlusion location in acute stroke with performance superior to that of 3D-TOF and gradient-echo T2 sequences.

ABBREVIATIONS: ExCICA = cervical/extracranial ICA; GRE T2 = gradient-echo T2; IICA = intrapetrous plus intracavernous segment; IVT = intravenous thrombolysis; MET = mechanical endovascular therapy; SAA = supra-aortic artery; SCICA = supraclinoid segment; TR-CE MRA = time-resolved contrast-enhanced MRA

$\mathbf{P}$ recisely identifying occlusion location on initial imaging of acute cerebral ischemic stroke is a key challenge for establishing prognosis and the most appropriate therapeutic management approach. Intravenous thrombolysis (IVT) efficacy is lower for proximal than distal occlusions, ranging from $8.7 \%$ for $\mathrm{ICA}^{1}$ to around $13 \%-30 \%$ for proximal MCA occlusions. ${ }^{2}$ Accurate identification of occlusion location also enables candidate selection

Received September 12, 2014; accepted after revision December 21.

From the Departments of Neuroradiology (A.L.B., H.R., J.-C.F., J.-Y.G.) and Neurology (T.R.), Centre Hospitalier Universitaire Rennes, Rennes, France; and Unité VISAGE U746 INSERM-INRIA, IRISA UMR CNRS 6074 (H.R., J.-C.F., J.-Y.G.), University of Rennes, Rennes, France.

This work was supported by the Comité de la Recherche Clinique, Centre Hospitalier Universitaire Rennes (contact grant LOC/10-11).

Please address correspondence to A. Le Bras, MD, Department of Neuroradiology, 2 Rue Henri Le Guilloux, 35033 Rennes, France; e-mail:

anthony.le.bras@chu-rennes.fr

三 Indicates article with supplemental on-line table.

http://dx.doi.org/10.3174/ajnr.A4264 for mechanical endovascular therapy (MET), which may be offered for large intracranial artery occlusions. ${ }^{3-5}$

Digital subtraction angiography remains the criterion standard of imaging techniques for precisely determining occlusion location. When MR imaging is used as the first-line technique in patients with acute stroke, $3 \mathrm{D}-\mathrm{TOF}$ is the recommended MRA sequence for depicting the level of intracranial occlusion. Some studies have demonstrated that the extra time needed to perform this additional sequence does not adversely affect outcomes. ${ }^{6,7}$ However, despite high sensitivity and specificity $(85 \%-100 \%$ and $91 \%-100 \%$, respectively) in identifying the absence of blood flow, ${ }^{8-11} 3 \mathrm{D}-\mathrm{TOF}$ cannot provide direct visualization of the thrombus ${ }^{12}$ and may misidentify the occlusion location due to its lack of sensitivity to slow flow. ${ }^{13,14}$ The gradient-echo T2 (GRE T2) sequence provides additional data at $77 \%-83 \%$ sensitivity for intracranial thrombus identification. ${ }^{15-17}$ Nevertheless, it may also misidentify the occlusion location because the associated susceptibility vessel sign depends on thrombus composition. Finally, none of these sequences provides supra-aortic artery (SAA) as- 


\begin{tabular}{lccc}
\hline & TR-CE MRA & 3D-TOF & GRE T2 \\
\hline Receive coil & Body coil & 8 -Channel brain coil & 8 -Channel brain coil \\
TR/TE (ms) & $4.0 / 1.25$ & $23 / 3.5$ & $757 / 16.1$ \\
Flip angle & $25^{\circ}$ & $15^{\circ}$ & $18^{\circ}$ \\
Acquisition plane & Coronal & Axial & Axial \\
FOV (mm) & $320 \times 320$ & $200 \times 200$ & $230 \times 230$ \\
Acquisition matrix & $268 \times 267$ & $312 \times 206$ & $512 \times 512$ \\
Section thickness (after interpolation) & $2.6(1.3)$ & $1.6(0.8)$ & 5 \\
Acquired voxel size $(\mathrm{mm})$ & $1.2 \times 1.2 \times 2.6$ & $0.64 \times 0.97 \times 1.6$ & $0.45 \times 0.45 \times 5$ \\
Reconstructed voxel size (mm) & $0.8 \times 0.8 \times 1.3$ & $0.39 \times 0.39 \times 0.8$ & 24 \\
No. of sections & 77 & 87 & - \\
Anteroposterior coverage (mm) & 100 & - & - \\
k-t BLAST undersampling factor & 4 & 1 & - \\
No. of dynamics & 12 & - & \\
Phase acquisition times & 8.7 sec & 2 minutes 36 seconds & \\
Total acquisition times & 1 minute 44 seconds & minute 37 seconds \\
\hline
\end{tabular}

sessment, though some of the recent grade A recommendations highlighted its utility in steno-occlusive disease screening for etiologic evaluation and subsequent appropriate endovascular therapy. ${ }^{18,19}$

Contrast-enhanced MRA can improve intracranial artery and SAA analysis. With improved contrast and less sensitivity to flow dynamics compared with 3D-TOF, this technique achieves optimal performance in detecting arterial diseases ${ }^{20-22}$ and is being increasingly used in conjunction with brain imaging techniques for acute stroke cases. ${ }^{14}$ However, contrast-enhanced MRA is limited because it provides only a single static acquisition restricted to the early arterial phase, and slow-flow dynamics can cause pseudo-occlusions to appear upstream of the steno-occlusive lesion. ${ }^{23,24}$ Recent time-resolved contrast-enhanced MRA (TR-CE MRA) sequences appear to come closest to DSA imaging used in depicting arteriovenous malformations or dural arteriovenous fistulas ${ }^{25,26}$ and theoretically take advantage of arterial filling dynamics, ${ }^{27}$ with the option of late phases, thus avoiding the lack of contrast filling of cervical arteries upstream of the intracranial occlusion.

This prospective study sought to assess the diagnostic performance of 3D-TOF, GRE T2, and TR-CE MRA in the identification of occlusion location, compared with DSA as the standard reference, in patients referred for MET in the acute phase of stroke.

\section{MATERIALS AND METHODS Patient Population}

We obtained both institutional review board approval and informed consent for this study, which complied with the Health Insurance Portability and Accountability Act. From August 2010 to December 2012, 33 consecutive patients who fulfilled the eligibility criteria were prospectively enrolled.

The inclusion criteria were as follows: age older than 18 years, MR imaging diagnosis of acute cerebral ischemic stroke based on diffusion imaging, referral for MET within 6 hs of symptom onset for anterior circulation occlusion, proximal thrombus detected by MR imaging, and time between MRA sequences and DSA $<3$ hs 30 minutes. The exclusion criteria included hemorrhagic lesions that were visible on MR images or comorbidity with poor prognosis.
MET was performed for every patient. For eligible patients, IVT was completed before endovascular thrombectomy, either immediately after IVT (combined therapy) or $1 \mathrm{~h}$ after IVT initiation if no clinical improvement was observed (rescue therapy). ${ }^{28}$

\section{MR Imaging}

Data acquisition was performed on a 3T MR imaging system (Achieva; Philips Healthcare, Best, the Netherlands), which is the first-line imaging technique available $24 / 7$ in our center. It was therefore possible to initiate optimal management without delay. ${ }^{12}$ The imaging protocol consisted of parenchymal brain imaging sequences (axial DWI, GRE T2, and FLAIR) and angiographic sequences (3D-TOF and TR-CE MRA). The MRA acquisition parameters are provided in Table 1 . The total average acquisition time for all MR imaging sequences was 12 minutes.

The TR-CE MRA sequence was based on a k-t Broad-use Linear Acquisition Speed-up Technique (BLAST)-accelerated 3D sequence, performed without moving the patient, with a wholebody birdcage coil used as a receiver. ${ }^{29,30}$ This technique provided wide coverage, from the aortic arch to the M3-M4 segments of the MCAs, with high temporal and spatial resolution. ${ }^{30}$ The acquisition slabs were positioned by using a low-resolution phase-contrast MRA that had been performed earlier. A fixed volume of a $15-\mathrm{mL}$ bolus of gadoterate meglumine (Dotarem; Guerbet, Aulnay-sous-Bois, France) was administered intravenously at 1.5 $\mathrm{mL} / \mathrm{s}$ with an automatic Spectris power injector (Medrad, Indianola, Pennsylvania), followed by a $30-\mathrm{mL}$ saline flush, without a bolus-tracking acquisition. Maximum-intensity-projection images of angiographic sequences were automatically generated, as with 3D-TOF.

\section{DSA Imaging}

Diagnostic prethrombectomy DSA was performed as the standard of reference for SAA and intracranial artery analysis. All DSA examinations were performed by a neurointerventionalist with $>5$ years' experience on a biplanar system (Allura Xper; Philips Healthcare) with the patient under general anesthesia by using the femoral artery approach. Angiographic images were acquired at 2 frames per second by using an automatic iodinated contrast medium injector at $6-8 \mathrm{~mL} / \mathrm{s}$ for intracranial arteries and $2-4 \mathrm{~mL} / \mathrm{s}$ for SAA (fluoroscopic guidance). 


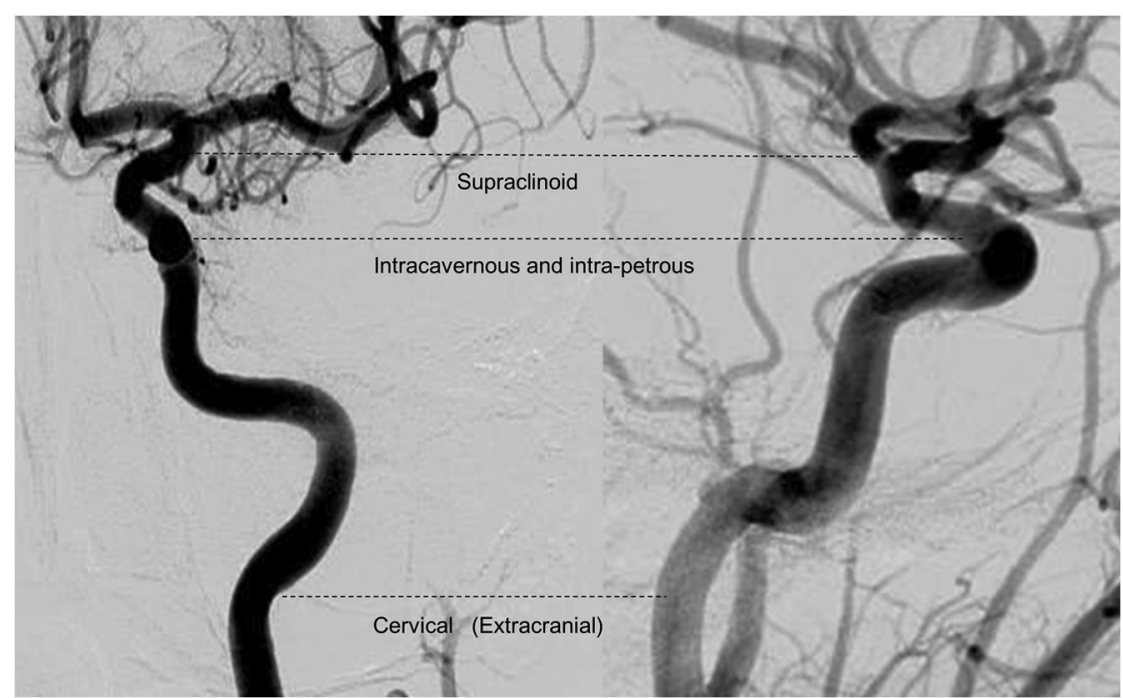

FIG 1. ICA segmentation used for the occlusion-level definition derived from Gibo et al, $1981{ }^{31}$ The internal carotid artery is divided into supraclinoid ICA, intracavernous and intrapetrous ICA, and cervical/extracranial ICA.

\section{Image Analysis}

The 3D-TOF, GRE T2, and TR-CE MRA sequences were reviewed on a specially designed imaging workstation (Extended MR WorkSpace 2.6.3; Philips Healthcare), which displayed native images and 3D MPR and MIP images. Two neuroradiologists, J.-C.F. and H.R., with 10 and 5 years' experience, respectively, were blinded to the DSA and clinical findings to independently evaluate the 3D-TOF and TR-CE MRA sequences. Any disagreements were discussed to reach a consensus. DSA was evaluated by 1 observer blinded to the other findings, by using frontal and lateral series on the target artery to define the standard of reference for the intracranial occlusion location and SAA stenosis quantification when applicable. In the event of tandem occlusion, both occlusion levels were reported. Patients were studied in randomized order. The diagnostic performance of both MRA sequences (TR-CE MRA and 3D-TOF) and the GRE T2 sequence was assessed and compared with DSA in terms of intracranial occlusion location. The diagnostic performance of TR-CE MRA was assessed and compared with DSA with regard to SAA stenosis detection. The images were assessed in terms of diagnostic performance and image quality.

The location of the intracranial occlusion caused by the thrombus was defined as follows: for both MRA (3D-TOF and TR-CE) sequences, the site of complete stoppage of the arterial flow; and for the GRE T2 sequence, the proximal portion of the susceptibility vessel sign. The ICA segmentation used for occlusion location was defined according to previous classifications, ${ }^{31,32}$ giving rise to the cervical/extracranial segment (ExCICA), intrapetrous plus intracavernous segment (IICA), and supraclinoid segment (SCICA) (Fig 1). The M1 and M2 segments of the MCA were defined by standard practice guidelines. Supraaortic artery stenosis was classified according to the semiquantitative SAA stenosis severity scale that is typically used in symptomatic patients, ${ }^{33,34}$ namely, none or moderate (stenosis, $<50 \%$ ), severe (stenosis, $>50 \%$ ), and occluded (complete stoppage of the arterial flow without downstream flow).

The contribution of the dynamic nature of TR-CE MRA was

\section{Statistical Analysis}

\section{RESULTS} was $120 \pm 33$ minutes. assessed by quantifying the number of phases that elapsed between the phase yielding maximum signal intensity within the nonpathologic arteries and the phase yielding maximum signal intensity directly upstream of the occlusion location.

The image diagnostic confidence index of both MRA sequences for SAA and intracranial arteries (Fig 1) was evaluated according to a modified diagnostic confidence index scale ${ }^{27,30,35}$ : nondiagnostic (vessel borders not definable), fair (vessel borders scarcely definable, inhomogeneous vessel signal), good (good delineation of vessel borders with slight irregularities, homogeneous vessel signal with slight artifacts), and excellent (sharp and complete delineation of vessel borders, homogeneous vessel signal without artifacts).

The statistical analysis was performed by using SPSS software (IBM, Armonk, New York). After recoding categoric variables as ordinal variables, we evaluated occlusion level differences with MRA sequences and GRE T2 compared with the reference imaging standard (DSA) by using a 2-tailed Wilcoxon exact signed rank test, with a statistically significant difference assumed for $P<$ .05 . To quantify the intermodality agreement of MRA sequences, comparing GRE T2 with DSA, we calculated the $\kappa$ coefficient, 95\% confidence intervals, and percentage agreement.

Finally, interobserver agreement for MRA image diagnostic confidence indexes for the major intracranial arteries was determined.

Of the 33 patients initially included in this study, 1 patient was later excluded due to a technical problem that occurred on gadolinium administration during the TR-CE MRA sequence, resulting in nonopacification of the SAA and intracranial arteries. The final number of patients included was 32 (On-line Table), with 21 men and 11 women (mean age, $64.9 \pm 14.8$ years; age range, $29-88$ years). This number included 19 (59.3\%) who had previously undergone IVT, 12 of whom had MET as a rescue therapy (if no substantial clinical improvement was seen $1 \mathrm{~h}$ after IVT). ${ }^{28}$ Two of these patients had a tandem occlusion. The mean time between MR imaging and DSA was $126 \pm 35$ minutes. For patients who underwent IVT, the mean time between IVT and DSA

\section{Intracranial Occlusion Location}

Artery occlusion was diagnosed for all patients on the basis of the 3D-TOF and TR-CE MRA sequences, whereas GRE T2 only provided a diagnosis in 26 of the 32 patients (81.2\%). Interobserver agreement for occlusion location was considered good for TR-CE MRA $(\kappa=0.72 ; 95 \%$ CI, 0.53-0.91) and excellent for 3D-TOF $(\kappa=0.86 ; 95 \%$ CI, 0.62-1).

A 2-tailed Wilcoxon exact signed rank test revealed significant AJNR Am J Neuroradiol 36:1081-88 Jun 2015 www.ajnr.org 
Table 2: Identification of intracranial occlusion location with 3D-TOF, GRE T2, and TR-CE MRA, compared with DSA as the standard reference $^{\mathrm{a}}$

\begin{tabular}{lcccc}
\hline \multicolumn{1}{c}{ Occlusion Location } & DSA (No.) (\%) & 3D-TOF MRA (No.) (\%) & GRE T2 (No.) (\%) & TR-CE MRA (No.) (\%) \\
\hline No occlusion (no susceptibility vessel & $0(0 \%)$ & $0(0 \%)$ & $6(17.6 \%)$ & $0(0 \%)$ \\
$\quad$ sign for GRE T2) & $3(8.8 \%)$ & $5(14.7 \%)$ & $0(0 \%)$ & $3(8.8 \%)$ \\
ExCICA & $0(0 \%)$ & $5(14.7 \%)$ & $0(0 \%)$ & $0(0 \%)$ \\
IICA & $8(23.5 \%)$ & $3(8.8 \%)$ & $3(8.8 \%)$ & $9(26.5 \%)$ \\
SCICA & $17(50.0 \%)$ & $20(58.8 \%)$ & $22(64.7 \%)$ & $19(55.9 \%)$ \\
M1 & $6(17.6 \%)$ & $1(2.9 \%)$ & $2(5.9 \%)$ & $3(8.8 \%)$ \\
M2 & & 0.43 & 0.31 & 0.81 \\
$\kappa$ & & $0.26-0.60$ & $0.15-0.48$ & $0.60-1$ \\
$95 \%$ Cl & & $61.8 \%$ & $55.9 \%$ & $88.2 \%$ \\
(\% of agreement) & & & \\
\hline
\end{tabular}

${ }^{a}$ Intracranial occlusion location with 3D-TOF, GRE T2, and TR-CE MRA, compared with DSA as the standard reference, showing the occlusion level detected as well as $\kappa$ and percentage of agreement with DSA for each MRI sequence.
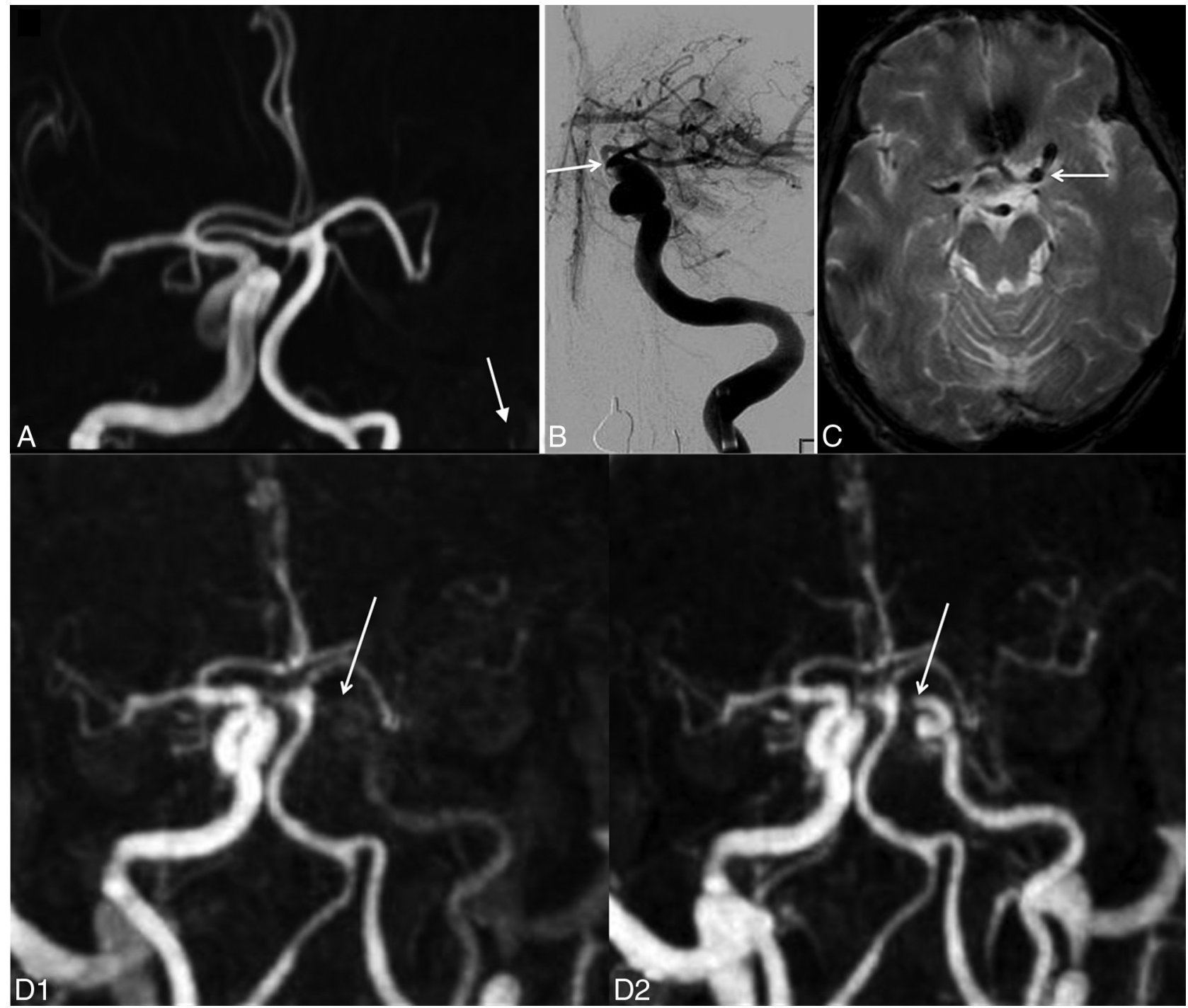

FIG 2. In patient 11, agreement between TR-CE MRA and DSA and discordance with TOF. A, 3D-TOF frontal MIP image shows complete occlusion of the left ICA from the IICA to distal segments of the left MCA. No data were available on the patency of the ICA proximal to the IICA segment (white arrow). B, Frontal DSA shows opacification of the left ICA up to the supraclinoid segment (white arrow). C, GRE T2 axial image displays a susceptibility vessel sign in the M1 segment of the left MCA. D, Frontal MIP TR-CE MRA shows patency of the left ICA up to the SCICA in the last phases (white arrows).

differences in the occlusion level among 3D-TOF, GRE T2, and DSA $(P<.001$ and $P=.002$, respectively), whereas there was no significant difference between DSA and TR-CE MRA $(P=.125)$.

Agreement between both MRA sequences and the GRE T2 sequence with DSA is shown in Table 2. The primary discrepancies between 3D-TOF and DSA were found when the occlusion was within the SCICA or M1 segments when using DSA, as opposed to the more proximal location within the IICA or even 


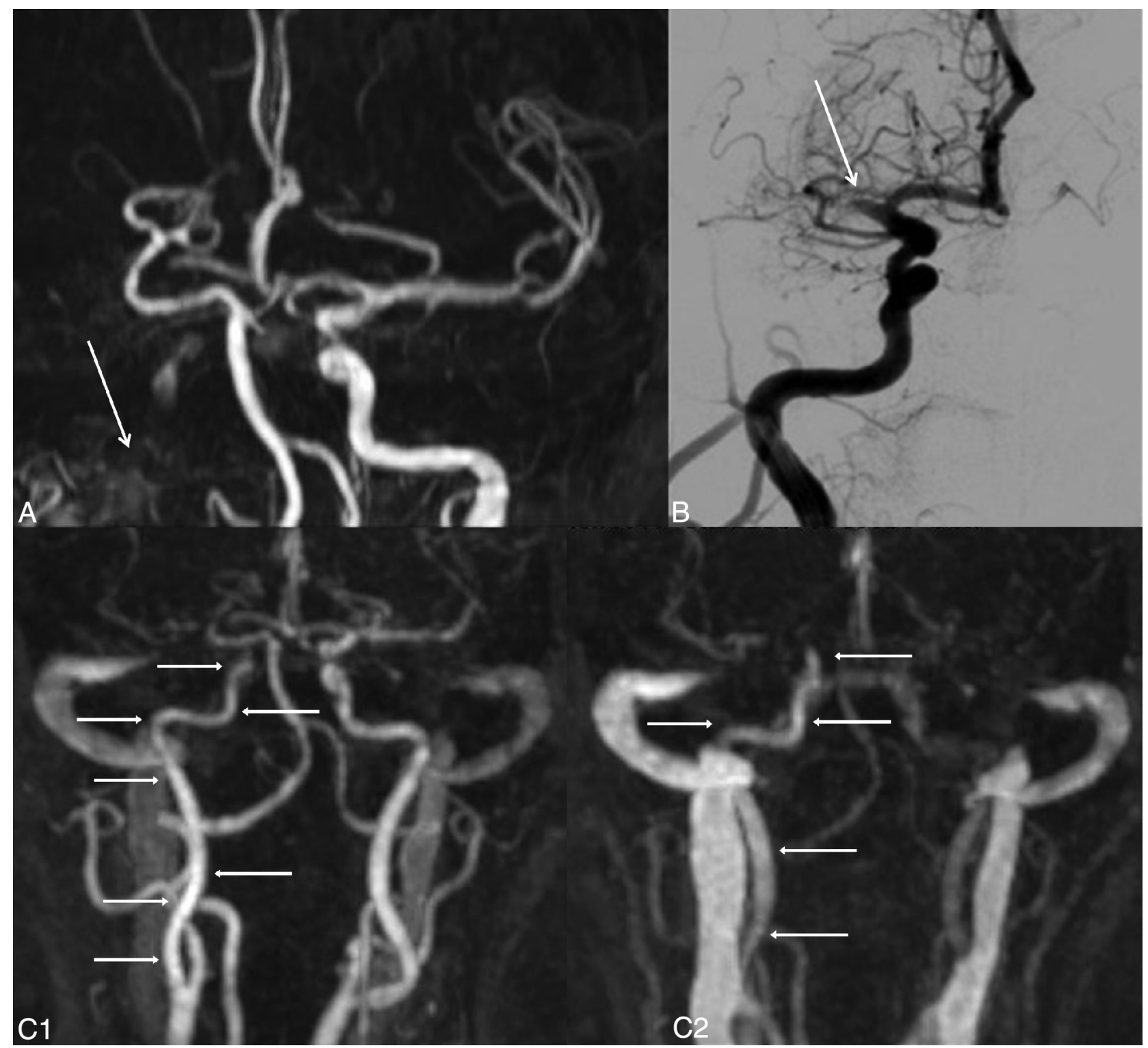

FIG 3. In patient 1 , disagreement between TR-CE MRA and DSA and discordance with TOF. A, 3D-TOF frontal MIP image shows complete occlusion of the right ICA from the IICA to distal segments of the left MCA, with a doubt about the patency of the ICA proximal to the IICA segment (white arrow). B, Frontal DSA shows opacification of the right ICA up to the Ml segment (white arrow). C, Frontal MIP TR-CE MRA shows patency of the right ICA up to the SCICA in the last phases (white arrows).

cervical/extracranial segment seen with 3D-TOF (Fig 2). The few discrepancies observed between TR-CE MRA and DSA occurred when the occlusion was within the M1 or M2 segments of the MCA when using DSA, with TR-CE MRA showing the occlusion more within the M1 segment of the MCA or in the SCICA (Fig 3).

\section{SAA Stenosis Detection}

SAA stenosis was classified with DSA as follows: none or moderate in 28 patients $(87.5 \%)$, severe in $1(3.1 \%)$, and occlusion in 3 (9.4\%). All cases were correctly classified on TR-CE MRA imaging (Fig 4).

\section{Dynamic Nature of TR-CE-MRA}

For 11 of the 32 patients (34.4\%), there was a time difference of at least 1 phase between maximum opacification of nonpathologic arteries and optimum opacification within the occluded segment. The difference was 1 phase for 6 patients, 2 phases for 3 patients, 3 phases for 1 patient, and 6 phases for 1 other patient. In 9 of these 11 patients $(81.8 \%)$, the occlusion location was misidentified on 3D-TOF, in contrast to only 2 misidentified cases with TR-CE MRA.

\section{Image Diagnostic Confidence Index}

For intracranial artery evaluation, the diagnostic confidence index was significantly higher $(P=.013)$ with the $3 \mathrm{D}$-TOF sequences (mean, $3.0 \pm 0.7$ ) than with the TR-CE MRA sequences (mean, $2.6 \pm 0.7$ ).

Interobserver agreement was considered good for the intracranial arteries visualized with the TR-CE MRA and 3D-TOF sequences $(\kappa=0.72 ; 95 \% \mathrm{CI}, 0.48-0.96$; and $\kappa=0.75$; $95 \%$ CI, 0.53-0.97, respectively) and moderate for SAAs visual- 


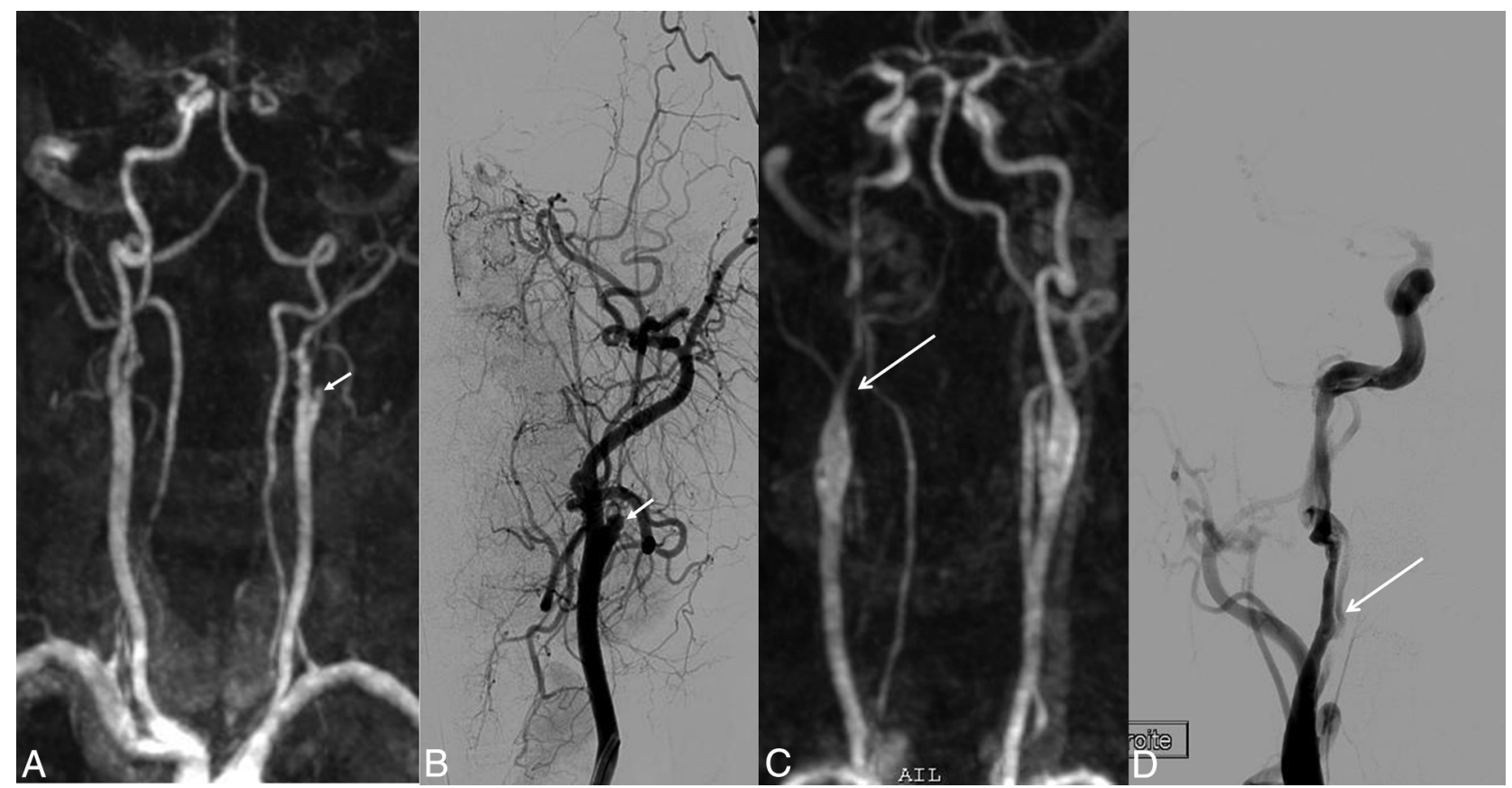

FIG 4. Examples of occlusion and stenosis of the extracranial arteries.TR-CE MRA and its corresponding frontal DSA image. In patient 7 ( $A$ and $B$ ), occlusion of the left ICA, probably of atheromatous etiology (white arrow) on TR-CE MRA $(A)$ and DSA (B). In patient $24(C$ and $D)$, severe stenosis ( $>50 \%$ ) of the right ICA, on TR-CE MRA $(C)$ and DSA (D), presumed due to an arterial dissection (white arrow).

ized with the TR-CE MRA sequences $(\kappa=0.41 ; 95 \% \mathrm{CI}$, $0.20-0.62)$.

\section{DISCUSSION}

Our study showed TR-CE MRA to be more accurate than 3DTOF and GRE T2 for identifying occlusion location, producing excellent agreement with DSA and offering a simultaneous and reliable SAA analysis with no additional time acquisition if substituted for the 3D-TOF sequence and entailing no delay in initiating optimal management.

The 3D-TOF showed low reliability for occlusion location, illustrated by its low $62 \%$ agreement with DSA $(\kappa=0.43)$. However, its sensitivity for thrombus detection was in line with the literature (100.0\%). ${ }^{8,10}$ Previous studies have already reported the limitation of 3D-TOF in terms of occlusion depiction, ${ }^{10,22}$ with Korogi et al, ${ }^{10}$ for example, demonstrating that $21 \%$ of the intracranial segments occluded in the 3D-TOF sequence proved permeable in the contrast-enhanced 3D-TOF sequence. In our study, despite using 3T MR imaging with an optimal signal-to-noise ratio for $3 \mathrm{D}-\mathrm{TOF}$ image quality, the discrepancies between the DSA and 3D-TOF sequences resulted in major misdiagnosis, especially when the occlusion was located in the ICA supraclinoid segment. The 3D-TOF sequence showed a tendency to locate the occlusion in a more proximal position than the DSA-located occlusions. This could be accounted for by the flow reduction upstream of the thrombus being erroneously detected by this flowbased technique. Similarly, GRE T2 achieved only 56\% agreement with DSA $(\kappa=0.31)$, despite its expected high sensitivity for thrombus detection $(81.2 \%) .{ }^{15-17}$

In contrast, TR-CE MRA proved a reliable non-time-consuming tool for occlusion location, with $88 \%$ agreement with DSA $(\kappa=0.81)$. The superior diagnostic performance of TR-CE MRA compared with 3D-TOF was not hampered by the lower image quality, probably due to the poorer spatial resolution, and may be the result of several factors: First, the T1 effect of gadolinium compensates for the signal loss caused by spin-dephasing that occurs in 3D-TOF when the flows are slow, turbulent, or oriented parallel to the section plane, in this case essentially upstream of the real occlusion site diagnosed on the DSA (standard of reference) image and especially within the IICA segment. Second, during the last phases, the dynamic nature of TR-CE MRA enables identification of the persistent slow-filling artery upstream of and closest to the occlusion. This flow can be missed on 3D-TOF, due to spin-dephasing, ${ }^{13}$ as well as on conventional CE-MRA, on account of only a single static arterial phase being available, a problem that has already been reported with $\mathrm{CT}$ in the setting of cerebral stroke. ${ }^{23,24}$ Both of these static conventional MRA contrast-enhanced or non-contrast-enhanced sequences, therefore, carry the risk of occlusion overestimation.

The advantage of dynamic imaging, as previously suggested in a study based on $4 \mathrm{D} \mathrm{CT},{ }^{36}$ was especially evident in our study. In some cases, we found that TR-CE MRA enabled rectification of the occlusion level that had been misdiagnosed on 3D-TOF due to its added advantage of late phases. Finally, the comparison of TR-CE MRA with the DSA technique in this study also confirmed its reliability for detecting SAA disease, a result that was previously reported. ${ }^{30}$ The ability of TR-CE MRA to provide simultaneous accurate data in terms of intracranial thrombus location and SAA stenosis during the initial MR imaging in a short acquisition time ( $<2$ minutes) is of particular significance. This advantage clearly means that TR-CE MRA may have the potential to improve patient triage and MET strategy planning. For example, identification of a tandem occlusion enables optimum MET preparation 
through the use of a triaxial guide-catheter system for stent placement as needed. ${ }^{37}$ Moreover, advanced knowledge of a critical case as tandem or a very proximal intracranial occlusion can determine the choice of general over local anesthesia. On the other hand, a single M1 occlusion can lead to single-catheter access and local anesthesia, thus resulting in a shorter procedure. ${ }^{38}$ Last, although a more distal occlusion level in M2 or further is not recommended for MET, overestimation of the level of occlusion (in M1 or more proximally) caused by $3 \mathrm{D}-\mathrm{TOF}$ may lead to the wrong therapeutic decision.

Our study has several limitations. First, due care should be taken when interpreting the results, given the moderate patient sample size, and a larger population should be assessed to confirm our data. Second, our study focused solely on the anterior circulation, and the presumed advantage of applying TR-CE MRA to the cerebral posterior circulation requires further investigation. Third, there was a time lapse between MR imaging and DSA. During this interval, arterial occlusion may spread or decrease regardless of ongoing thrombosis or fibrinolysis processes, resulting in discrepancies in the occlusion location between MR imaging and DSA. However, the high rate of concordance reported between TR-CE MRA and DSA suggests that this limitation did not influence our conclusions. Fourth, we did not compare TRCE MRA with conventional contrast-enhanced MRA because it would have required time and a second injection; also, the agreement between the 2 sequences had been demonstrated previously. ${ }^{30}$ Finally the TR-CE MRA sequence has a low spatial resolution, which obviously reduces image quality for intracranial arteries and hampers analysis beyond the M2 or M1 segments in some cases. This drawback could be easily remedied by using dedicated coils and parallel imaging. ${ }^{39,40}$

\section{CONCLUSIONS}

Of all the MR imaging sequences we evaluated, TR-CE MRA proved to be the most reliable for identifying occlusion location in the setting of acute stroke, offering superior performance to 3D-TOF and T2 GRE, along with the added advantage of enabling SAA stenosis detection. Thus TR-CE MRA could replace $3 \mathrm{D}-\mathrm{TOF}$ and so reduce the interval before optimal management, especially when endovascular treatment is considered. The GRE T2 sequence remains mandatory because it enables hemorrhage identification.

\section{ACKNOWLEDGMENTS}

The authors thank Aymeric Stamm, MD, for supervising the statistical analysis of this study.

\section{REFERENCES}

1. del Zoppo GJ, Poeck K, Pessin MS, et al. Recombinant tissue plasminogen activator in acute thrombotic and embolic stroke. Ann Neurol 1992;32:78-86

2. Bhatia $\mathrm{R}, \mathrm{Hill} \mathrm{MD}$, Shobha $\mathrm{N}$, et al. Low rates of acute recanalization with intravenous recombinant tissue plasminogen activator in ischemic stroke: real-world experience and a call for action. Stroke 2010;41:2254-58

3. Mazighi M, Serfaty JM, Labreuche J, et al. Comparison of intravenous alteplase with a combined intravenous-endovascular approach in patients with stroke and confirmed arterial occlusion
(RECANALISE study): a prospective cohort study. Lancet Neurol 2009;8:802-09

4. Vendrell JF, Mernes R, Nagot N, et al. Evaluation of an intravenousendovascular strategy in patients with acute proximal middle cerebral artery occlusion. AJNR Am J Neuroradiol 2013;34:603-08

5. Leker RR, Eichel R, Gomori JM, et al. Stent-based thrombectomy versus intravenous tissue plasminogen activator in patients with acute middle cerebral artery occlusion. Stroke 2012;43:3389-91

6. Breuer L, Schellinger PD, Huttner HB, et al. Feasibility and safety of magnetic resonance imaging-based thrombolysis in patients with stroke on awakening: initial single-centre experience. Int J Stroke 2010;5:68-73

7. Köhrmann M, Schellinger PD. Stroke-MRI: extending the timewindow: recent trials and clinical practice. Int J Stroke 2007;2:53-54

8. Stock KW, Radue EW, Jacob AL, et al. Intracranial arteries: prospective blinded comparative study of MR angiography and DSA in 50 patients. Radiology 1995;195:451-56

9. Stock KW, Wetzel S, Kirsch E, et al. Anatomic evaluation of the circle of Willis: MR angiography versus intraarterial digital subtraction angiography. AJNR Am J Neuroradiol 1996;17:1495-99

10. Korogi $Y$, Takahashi $M$, Mabuchi $N$, et al. Intracranial vascular stenosis and occlusion: diagnostic accuracy of three-dimensional, Fourier transform, time-of-flight MR angiography. Radiology 1994;193:187-93

11. Hirai T, Korogi Y, Ono K, et al. Prospective evaluation of suspected stenoocclusive disease of the intracranial artery: combined MR angiography and CT angiography compared with digital subtraction angiography. AJNR Am J Neuroradiol 2002;23:93-101

12. Wintermark $M$, Sanelli PC, Albers GW, et al. Imaging recommendations for acute stroke and transient ischemic attack patients: a joint statement by the American Society of Neuroradiology, the American College of Radiology, and the Society of NeuroInterventional Surgery. AJNR Am J Neuroradiol 2013;34:E117-27

13. Ishimaru $\mathrm{H}$, Ochi M, Morikawa M, et al. Accuracy of pre- and postcontrast $3 \mathrm{D}$ time-of-flight MR angiography in patients with acute ischemic stroke: correlation with catheter angiography. AJNR Am J Neuroradiol 2007;28:923-26

14. Sohn $\mathrm{CH}$, Sevick RJ, Frayne R. Contrast-enhanced MR angiography of the intracranial circulation. Magn Reson Imaging Clin $\mathrm{N} \mathrm{Am}$ 2003;11:599-614

15. Flacke S, Urbach H, Keller E, et al. Middle cerebral artery (MCA) susceptibility sign at susceptibility-based perfusion MR imaging: clinical importance and comparison with hyperdense MCA sign at CT 1. Radiology 2000;215:476-82

16. Rovira A, Orellana P, Alvarez-Sabín J, et al. Hyperacute ischemic stroke: middle cerebral artery susceptibility sign at echo-planar gradient-echo MR imaging. Radiology 2004;232:466-73

17. Naggara O, Raymond J, Domingo Ayllon M, et al. T2* “susceptibility vessel sign" demonstrates clot location and length in acute ischemic stroke. PLoS One 2013;8:e76727

18. Latchaw RE, Alberts MJ, Lev MH, et al. Recommendations for imaging of acute ischemic stroke: a scientific statement from the American Heart Association. Stroke 2009;40:3646-78

19. Jauch EC, Saver JL, Adams HP, et al. Guidelines for the early management of patients with acute ischemic stroke: a guideline for healthcare professionals from the American Heart Association/ American Stroke Association. Stroke 2013;44:870-947

20. Anzalone N, Scomazzoni F, Castellano R, et al. Carotid artery stenosis: intraindividual correlations of $3 \mathrm{D}$ time-of-flight $\mathrm{MR}$ angiography, contrast-enhanced MR angiography, conventional DSA, and rotational angiography for detection and grading. Radiology 2005;236:204-13

21. Yang CW, Carr JC, Futterer SF, et al. Contrast-enhanced MR angiography of the carotid and vertebrobasilar circulations. AJNR Am J Neuroradiol 2005;26:2095-101

22. Yang JJ, Hill MD, Morrish WF, et al. Comparison of pre- and postcontrast 3D time-of-flight MR angiography for the evaluation of 
distal intracranial branch occlusions in acute ischemic stroke. AJNR Am J Neuroradiol 2002;23:557-67

23. Kim JJ, Dillon WP, Glastonbury CM, et al. Sixty-four-section multidetector CT angiography of carotid arteries: a systematic analysis of image quality and artifacts. AJNR Am J Neuroradiol 2010;31:91-99

24. Schreiber S, Schreiber F, Glaser M, et al. Detecting artery occlusion and critical flow diminution in the case of an acute ischemic stroke: methodological pitfalls of common vascular diagnostic methods. Ultraschall Med 1980 2011;32:274-80

25. Meckel S, Maier M, Ruiz DSM, et al. MR angiography of dural arteriovenous fistulas: diagnosis and follow-up after treatment using a time-resolved 3D contrast-enhanced technique. AJNR Am J Neuroradiol 2007;28:877-84

26. Unlu E, Temizoz O, Albayram S, et al. Contrast-enhanced MR 3D angiography in the assessment of brain AVMs. Eur J Radiol 2006;60:367-78

27. Raoult H, Ferré JC, Morandi X, et al. Quality-evaluation scheme for cerebral time-resolved 3D contrast-enhanced MR angiography techniques. AJNR Am J Neuroradiol 2010;31:1480-87

28. Costalat V, Machi P, Lobotesis K, et al. Rescue, combined, and standalone thrombectomy in the management of large vessel occlusion stroke using the Solitaire device: a prospective 50-patient singlecenter study: timing, safety, and efficacy. Stroke 2011;42:1929-35

29. Kozerke S, Tsao J, Razavi R, et al. Accelerating cardiac cine 3D imaging using k-t BLAST. Magn Reson Med 2004;52:19-26

30. Ferré JC, Raoult H, Breil S, et al. Supra-aortic arteries: three-dimensional time-resolved k-t BLAST contrast-enhanced MRA using a nondedicated body coil at 3 Tesla in acute ischemic stroke. J Magn Reson Imaging 2014;40:1056-63

31. Gibo H, Lenkey C, Rhoton AL Jr. Microsurgical anatomy of the su- praclinoid portion of the internal carotid artery. I Neurosurg 1981;55:560-74

32. Shapiro $M, B e c s k e ~ T$, Riina HA, et al. Toward an endovascular internal carotid artery classification system. AJNR Am J Neuroradiol 2014;35:230-36

33. Hinkmann FM, Voit HL, Anders K, et al. Ultra-fast carotid CTangiography: low versus standard volume contrast material protocol for a 128-slice CT-system. Invest Radiol 2009;44:257-64

34. Rerkasem K, Rothwell PM. Carotid endarterectomy for symptomatic carotid stenosis. Cochrane Database Syst Rev 2011;CD001081

35. Kramer H, Runge VM, Morelli JN, et al. Magnetic resonance angiography of the carotid arteries: comparison of unenhanced and contrast enhanced techniques. Eur Radiol 2011;21:1667-76

36. Frölich AM, Schrader D, Klotz E, et al. 4D CT angiography more closely defines intracranial thrombus burden than single-phase CT angiography. AJNR Am J Neuroradiol 2013;34:1908-13

37. Machi P, Lobotesis K, Maldonado IL, et al. Endovascular treatment of tandem occlusions of the anterior cerebral circulation with Solitaire FR thrombectomy system: initial experience. Eur J Radiol 2012;81:3479-84

38. Soize S, Kadziolka K, Estrade L, et al. Mechanical thrombectomy in acute stroke: prospective pilot trial of the Solitaire FR device while under conscious sedation. AJNR Am J Neuroradiol 2013;34:360-65

39. Parikh PT, Sandhu GS, Blackham KA, et al. Evaluation of image quality of a 32-channel versus a 12-channel head coil at 1.5T for MR imaging of the brain. AJNR Am J Neuroradiol 2011;32:365-73

40. De Zwart JA, Ledden PJ, van Gelderen P, et al. Signal-to-noise ratio and parallel imaging performance of a 16-channel receive-only brain coil array at 3.0 Tesla. Magn Reson Med 2004;51:22-26 